\title{
3-Loop Heavy Flavor Corrections in Deep-Inelastic Scattering with Two Heavy Quark Lines
}

\author{
J. Ablinger ${ }^{a}$, J. Blümlein ${ }^{b}$, A. De Freitas ${ }^{b}$, A. Hasselhuhn ${ }^{a, b}$, A. von Manteuffel ${ }^{c}$, \\ M. Round ${ }^{a, b}$, C. Schneider ${ }^{a}$ and F. Wißbrock ${ }^{* a, b, d \dagger}$
}

${ }^{a}$ Research Institute for Symbolic Computation (RISC), Johannes Kepler University, Altenbergerstraße 69, A-4040 Linz, Austria

${ }^{b}$ Deutsches Elektronen-Synchrotron, DESY, Platanenallee 6, D-15738 Zeuthen, Germany

${ }^{c}$ PRISMA Cluster of Excellence, Institute of Physics, J. Gutenberg University Mainz, D-55099 Mainz,Germany

${ }^{d}$ Institut des Hautes Études Scientifiques, IHES, Route de Chartres 35, F-91440 Bures-sur-Yvette, France

E-mail: jablinge@risc.uni-linz.ac.at, Johannes.Bluemlein@desy.de, abilio.de.freitas@desy.de, alexander.hasselhuhn@desy.de, manteuffeleuni-mainz.de, mark.roundedesy.de, cschneiderisc.uni-linz.ac.at, fabian.wissbrock@desy.de

We consider gluonic contributions to the heavy flavor Wilson coefficients at 3-loop order in QCD with two heavy quark lines in the asymptotic region $Q^{2} \gg m_{1(2)}^{2}$. Here we report on the complete result in the case of two equal masses $m_{1}=m_{2}$ for the massive operator matrix element $A_{g g, Q}^{(3)}$, which contributes to the corresponding heavy flavor transition matrix element in the variable flavor number scheme. Nested finite binomial sums and iterated integrals over square-root valued alphabets emerge in the result for this quantity in $N$ and $x$-space, respectively. We also present results for the case of two unequal masses for the flavor non-singlet operator matrix elements and on the scalar integrals in case of $A_{g g, Q}^{(3)}$, which were calculated without a further approximation. The graphs can be expressed by finite nested binomial sums over generalized harmonic sums, the alphabet of which contains rational letters in the ratio $\eta=m_{1}^{2} / m_{2}^{2}$.

Loops and Legs in Quantum Field Theory - LL 2014,

April 27th-March 2nd 2014

Weimar, Germany

\footnotetext{
*Speaker.

${ }^{\dagger}$ DESY 14-121, DO-TH 14/12, MITP/14-047, SFB/CPP-14-36, LPN14-085
}

We would like to thank M. Steinhauser for the possibility to use the code $22 \mathrm{e} / \mathrm{EXP}$. This work was supported in part by DFG Sonderforschungsbereich Transregio 9, Computergestützte Theoretische Teilchenphysik, Studienstiftung des Deutschen Volkes, the Austrian Science Fund (FWF) grants P20347-N18 and SFB F50 (F5009-N15), the European Commission through contracts PITN-GA-2010-264564 (LHCPhenoNet) and PITN-GA-2012-316704 (HIGGSTOOLS), by the Research Center "Elementary Forces and Mathematical Foundations (EMG)" of J. Gutenberg University Mainz and DFG, and by FP7 ERC Starting Grant 257638 PAGAP. 


\section{Introduction}

Beginning with 3-loop order in QCD the heavy flavor contributions to the deep-inelastic structure functions, such as $F_{2}\left(x, Q^{2}\right)$, contain Feynman diagrams with two massive quark lines, either of equal or unequal mass. In the asymptotic region of large virtualities $Q^{2} \gg m^{2}$ for the former case, calculations of a series of Mellin moments [1] have been performed. It is well-known that the asymptotic picture agrees with the exact one at an accuracy of $O(1 \%)$ at next-to-leading order for scales $Q^{2} / m^{2} \approx 10$ for the structure function $F_{2}\left(x, Q^{2}\right)$ [2]. By now, the complete set of logarithmic contributions [3] is known and the massive Wilson coefficients and/or operator matrix elements

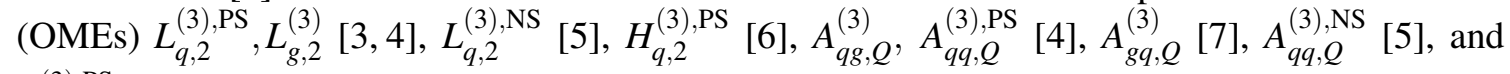
$A_{Q q}^{(3), \mathrm{PS}}[6]$ have been calculated completely. ${ }^{1}$

Since the ratio $m_{c}^{2} / m_{b}^{2} \sim 1 / 10$, with $m_{c}$ and $m_{b}$ the charm and bottom quark masses, is not small, the charm quark cannot be considered massless at the scale $\mu=m_{b}$. Therefore a sequential decoupling of these two heavy quark flavors using the single quark decoupling in the usual variable heavy flavor scheme (VFNS) $[1,8,9]$ is not possible ${ }^{2}$. Instead a generalization of the VFNS, decoupling two massive quarks at the same time, is necessary [11].

In the case of two different masses $m_{c}$ and $m_{b}$, one may derive the asymptotic heavy flavor Wilson coefficients as well by considering the region of virtualities $Q^{2} \gg m_{b}^{2}, m_{c}^{2}$, where power corrections $O\left(m_{c, b}^{2} / Q^{2}\right)$ can be neglected. The heavy flavor Wilson coefficients are known to factorize into the light flavor coefficients $C_{i,(2, L)}$ and the process-independent massive operator matrix elements (OMEs) $A_{i j}$, see Refs. [2,8] for the corresponding relations.

The massive OMEs are evaluated in terms of Feynman diagrams with additional Feynman rules for the composite operator insertions. The moments $N=2,4,6$ to all operator matrix elements have been calculated up to $O\left(\frac{m_{c}^{6}}{m_{b}^{6}} \ln ^{3}\left(m_{c}^{2} / m_{b}^{2}\right)\right)$ for the contributions containing both charm and bottom-lines [11-13] using the projection method [1] through which the OMEs are mapped to tadpoles. They have been calculated using the code 22 e/EXP [14]. This required a total computation time of about $1 \mathrm{CPU}$-year.

In this note we report on recent results on contributions to OMEs containing two massive fermion lines of equal and unequal quark masses. In Section 2 we discuss the contributions of $O\left(\alpha_{s}^{3} T_{F}^{2} C_{F(A)}\right)$ to the OME $A_{g g, Q}$ in the case of equal masses. The renormalization of the unequal mass case is briefly discussed in Section 3. There we also present results for the massive OME in the flavor non-singlet and transversity cases and for a scalar diagram with unequal masses at general Mellin variable $N$ and arbitrary mass ratio contributing to $A_{g g, Q}$. Section 4 contains the conclusions.

\section{The $O\left(\alpha_{s}^{3} T_{F}^{2}\right)$ Contributions to $A_{g g, Q}$}

The contributions of $O\left(\alpha_{s}^{3} T_{F}^{2} C_{F, A}\right)$ to the operator matrix element $A_{g g, Q}$ have been calculated in Ref. [15]. Here the color factors are $C_{F}=\left(N_{c}^{2}-1\right) /\left(2 N_{c}\right), C_{A}=N_{c}, T_{F}=1 / 2$ in $S U\left(N_{c}\right)$ and

\footnotetext{
${ }^{1}$ For the notation we refer to Ref. [1].

${ }^{2}$ The value of the decoupling scale in the VFNS is process dependent and usually not the scale of the decoupling heavy quark mass, cf. Ref. [10].
} 
$N_{c}=3$ for QCD. Most of the diagrams have been computed directly using Mellin-Barnes representations and generating function techniques directly, i.e. including the corresponding numerator structures. This led to large amounts of nested sums which were treated with the summation technologies encoded in the package Sigma [16,17], based on advanced symbolic summation algorithms in the setting of difference fields [18-26], and the packages EvaluateMultisums, SumProduction [27], and RhoSum [28], which are all based on Sigma. In part of the sums we used integration-by-parts reduction applying Reduze $2[29,30]^{3}$ and calculated the corresponding master integrals using differential equations and also applying Mellin-Barnes techniques.

As a main result of Ref. [15] we present the constant part to the unrenormalized operator matrix element $a_{g g, Q ; \mathrm{T}_{\mathrm{F}}^{2}}^{(3)}$. It is finally obtained by the following compact expression

$$
\begin{aligned}
& a_{g g, Q ; \mathrm{T}_{\mathrm{F}}^{2}}^{(3)}(N)= \\
& C_{F} T_{F}^{2}\left\{\frac{16}{27} F S_{1}^{3}+\frac{16 Q_{4}}{27(N-1) N^{3}(N+1)^{3}(N+2)} S_{1}^{2}+\left[-\frac{16}{3} F S_{2}\right.\right. \\
& \left.-\frac{32 Q_{10}}{81(N-1) N^{4}(N+1)^{4}(N+2)(2 N-3)(2 N-1)}\right] S_{1}-\frac{16 Q_{4}}{9(N-1) N^{3}(N+1)^{3}(N+2)} S_{2} \\
& -\frac{2 Q_{13}}{243(N-1) N^{5}(N+1)^{5}(N+2)(2 N-3)(2 N-1)}-F\left[\frac{352}{27} S_{3}-\frac{64}{3} S_{2,1}\right] \\
& +\left[\frac{16}{3} F S_{1}-\frac{8 Q_{8}}{9(N-1) N^{3}(N+1)^{3}(N+2)}\right] \zeta_{2}+\frac{Q_{3}}{9(N-1) N^{2}(N+1)^{2}(N+2)} \zeta_{3} \\
& \left.-\left(\begin{array}{c}
2 N \\
N
\end{array}\right) \frac{16 Q_{5}}{3(N-1) N(N+1)^{2}(N+2)(2 N-3)(2 N-1)} \frac{1}{4^{N}}\left(\sum_{i=1}^{N} \frac{4^{i} S_{1}(i-1)}{i^{2}\left(\begin{array}{c}
2 i \\
i
\end{array}\right)}-7 \zeta_{3}\right)\right\} \\
& +C_{A} T_{F}^{2}\left\{-\frac{4 Q_{2}}{135(N-1) N^{2}(N+1)^{2}(N+2)} S_{1}^{2}+\frac{16\left(4 N^{3}+4 N^{2}-7 N+1\right)}{15(N-1) N(N+1)}\left[S_{2,1}-S_{3}\right]\right. \\
& +\frac{Q_{12}}{3645(N-1) N^{4}(N+1)^{4}(N+2)(2 N-3)(2 N-1)} \\
& -\frac{8 Q_{11}}{3645(N-1) N^{3}(N+1)^{3}(N+2)(2 N-3)(2 N-1)} S_{1}+\frac{4 Q_{7}}{135(N-1) N^{2}(N+1)^{2}(N+2)} S_{2} \\
& -\left(\begin{array}{c}
2 N \\
N
\end{array}\right) \frac{4 Q_{9}}{45(N-1) N(N+1)^{2}(N+2)(2 N-3)(2 N-1)} \frac{1}{4^{N}}\left(\sum_{i=1}^{N} \frac{4^{i} S_{1}(i-1)}{i^{2}\left(\begin{array}{c}
2 i \\
i
\end{array}\right)}-7 \zeta_{3}\right) \\
& +\left[\frac{4 Q_{6}}{27(N-1) N^{2}(N+1)^{2}(N+2)}-\frac{560}{27} S_{1}\right] \zeta_{2}+\left[-\frac{7 Q_{1}}{270(N-1) N(N+1)(N+2)}\right. \\
& \left.\left.-\frac{1120}{27} S_{1}\right] \zeta_{3}\right\} \text {. }
\end{aligned}
$$

Here we define

$$
F(N)=\frac{\left(2+N+N^{2}\right)^{2}}{(N-1) N^{2}(N+1)^{2}(N+2)} \equiv F,
$$

\footnotetext{
${ }^{3}$ The package Reduze2 uses Fermat [31] and GiNac [32].
} 
and $Q_{i}$ denote polynomials in $N$, cf. [15]. The equal mass contribution to the massive OME $A_{g g, Q}^{(3)}$ can be described by harmonic sums $[33,34] S_{\vec{a}}(N) \equiv S_{\vec{a}}$,

$$
S_{b, \vec{a}}(N)=\sum_{k=1}^{N} \frac{(\operatorname{sign}(b))^{k}}{k^{|b|}} S_{\vec{a}}(k), \quad S_{\emptyset}=1, b, a_{i} \in \mathbb{Z} \backslash\{0\}
$$

and one inverse binomial sum weighted by a harmonic sum [35]. In $x$-space the latter sum results in an iterated integral of square root-valued letters, extending the space of functions for contributions to the 3-loop Wilson coefficients having been know so far for the first time.

\section{Scalar Integrals of the $O\left(\alpha_{s}^{3} T_{F}^{2}\right)$ Contributions to $A_{g g, Q}$ with Two Different Masses}

We have calculated all scalar integrals contributing to the OME $A_{g g, Q}$ in case of two different masses both in $x$ - and $N$-space, without any approximation, like e.g. an expansion in $\eta=m_{c}^{2} / m_{b}^{2}$. We first describe the main steps of the renormalization of these contributions, cf. Ref. [11] for details, present results in the flavor non-singlet case, and finally give an example for the scalar integrals contributing to $A_{g g, Q}^{(3)}$.

\subsection{Renormalization}

The renormalization of the operator matrix elements with two heavy quark flavors is performed as a generalization of the single mass case in Ref. [1]. Here the sub-set of graphs with two massive fermion lines is considered. It is technically of advantage to treat the equal and different mass cases together. The renormalization of the unequal mass case is then obtained by subtracting the contributions in the equal mass case taken from [1]. The quark mass is either renormalized in the on-shell renormalization scheme or the $\overline{\mathrm{MS}}$ scheme [36]. The factorization relation [2] at large virtualities strictly requires the external legs of the operator matrix elements to be on-shell. Charge renormalization is easiest carried out in a MOM-scheme applying the background field method [37]. Afterwards a finite renormalization to the $\overline{\mathrm{MS}}$-scheme is performed. The remaining ultraviolet singularities of the composite operators are renormalized via the corresponding $Z_{i j}$-factors and in a final step the collinear singularities are removed as they are absorbed into the parton distribution functions. In all the quantities, corrections due to the two mass scales $m_{c}$ and $m_{b}$ are emerging. Accordingly one may generalize the VFNS w.r.t. the simultaneous decoupling of both $m_{c}$ and $m_{b}$, which is needed from 3-loop order onwards, since here graphs with two heavy quark lines contribute. Their mass dependence does not factorize, unlike the case up to 2-loop order.

\subsection{Operator matrix elements with $m_{1} \neq m_{2}$}

In the case of a general Mellin variable $N$ the 3-loop graphs with two different masses have been calculated for the OME in the non-singlet and transversity cases and for general mass ratios $\eta$. Only a few Feynman diagrams contribute in these cases. Here we show the unequal mass contribution to the constant part of the unrenormalized OME, cf. [1], for the non-singlet and transversity cases

$$
a_{q q, Q}^{(3), \mathrm{NS}}=C_{F} T_{F}^{2}\left\{\left(\frac{32}{27} S_{1}-\frac{8\left(3 N^{2}+3 N+2\right)}{27 N(N+1)}\right) \ln ^{3}(\eta)+\left[-\frac{R_{1}}{18 N^{2}(N+1)^{2} \eta}\right.\right.
$$




$$
\begin{aligned}
& +\left[\frac{\left(3 N^{2}+3 N+2\right)(\eta+1)\left(5 \eta^{2}+22 \eta+5\right)}{36 N(N+1) \eta^{3 / 2}}-\frac{(\eta+1)\left(5 \eta^{2}+22 \eta+5\right)}{9 \eta^{3 / 2}} S_{1}\right] \ln \left(\frac{1+\eta_{1}}{1-\eta_{1}}\right) \\
& \left.+\frac{2\left(5 \eta^{2}+2 \eta+5\right)}{9 \eta} S_{1}+\ln (1-\eta)\left(\frac{16\left(3 N^{2}+3 N+2\right)}{9 N(N+1)}-\frac{64}{9} S_{1}\right)+\frac{32}{9} S_{2}\right] \ln ^{2}(\eta) \\
& +\left[\frac{40(\eta-1)(\eta+1)}{9 \eta} S_{1}-\frac{10\left(3 N^{2}+3 N+2\right)(\eta-1)(\eta+1)}{9 N(N+1) \eta}+\frac{(\eta+1)\left(5 \eta^{2}+22 \eta+5\right)}{9 \eta^{3 / 2}}\right. \\
& \times\left[8 S_{1}-\frac{2\left(3 N^{2}+3 N+2\right)}{N(N+1)}\right] \mathrm{Li}_{2}\left(\eta_{1}\right) \\
& \left.+\frac{\left(\eta_{1}+1\right)^{2}\left(-10 \eta^{3 / 2}+5 \eta^{2}+42 \eta-10 \eta_{1}+5\right)}{9 \eta^{3 / 2}}\left[\frac{\left(3 N^{2}+3 N+2\right)}{2 N(N+1)}-2 S_{1}\right] \operatorname{Li}_{2}(\eta)\right] \ln (\eta) \\
& +\frac{16\left(3 N^{4}+6 N^{3}+47 N^{2}+20 N-12\right) \zeta_{2}}{27 N^{2}(N+1)^{2}}+\frac{(\eta+1)\left(5 \eta^{2}+22 \eta+5\right)}{9 \eta^{3 / 2}}\left[\frac{4\left(3 N^{2}+3 N+2\right)}{N(N+1)}\right. \\
& \left.-16 S_{1}\right] \operatorname{Li}_{3}\left(\eta_{1}\right)+\frac{\left(\eta_{1}+1\right)^{2}\left(-10 \eta^{3 / 2}+5 \eta^{2}+42 \eta-10 \eta_{1}+5\right)}{9 \eta^{3 / 2}}\left[2 S_{1}\right. \\
& \left.-\frac{\left(3 N^{2}+3 N+2\right)}{2 N(N+1)}\right] \operatorname{Li}_{3}(\eta)+\left[\frac{16\left(405 \eta^{2}-3238 \eta+405\right)}{729 \eta}+\frac{256 \zeta_{3}}{27}-\frac{640 \zeta_{2}}{27}\right] S_{1} \\
& \left.+\left[\frac{128 \zeta_{2}}{9}+\frac{3712}{81}\right] S_{2}-\frac{1280}{81} S_{3}+\frac{256}{27} S_{4}-\frac{64\left(3 N^{2}+3 N+2\right) \zeta_{3}}{27 N(N+1)}-\frac{4 R_{2}}{729 N^{4}(N+1)^{4} \eta}\right\}
\end{aligned}
$$

and

$$
\begin{aligned}
a_{q q, Q}^{(3), \mathrm{NS}, \mathrm{TR}}= & C_{F} T_{F}^{2}\left\{\left[\frac{16}{27} S_{1}-\frac{4}{9}\right] \ln ^{3}(\eta)+\left[-\frac{(\eta+5)(5 \eta+1)}{12 \eta}+\frac{(\eta+1)\left(5 \eta^{2}+22 \eta+5\right)}{72 \eta^{3 / 2}}\left[4 S_{1}-3\right]\right.\right. \\
& \left.\times \ln \left(\frac{1-\eta_{1}}{1+\eta_{1}}\right)+\frac{\left(5 \eta^{2}+2 \eta+5\right)}{9 \eta} S_{1}+\ln (1-\eta)\left(\frac{8}{3}-\frac{32}{9} S_{1}\right)+\frac{16}{9} S_{2}\right] \ln ^{2}(\eta) \\
& +\left[\frac{20(\eta-1)(\eta+1)}{9 \eta} S_{1}-\frac{5(\eta-1)(\eta+1)}{3 \eta}+\frac{(\eta+1)\left(5 \eta^{2}+22 \eta+5\right)}{9 \eta^{3 / 2}}\left[4 S_{1}-3\right]\right. \\
& \left.\times \operatorname{Li}_{2}\left(\eta_{1}\right)+\frac{\left(\eta_{1}+1\right)^{2}\left(-10 \eta^{3 / 2}+5 \eta^{2}+42 \eta-10 \eta_{1}+5\right)}{36 \eta^{3 / 2}}\left[3-4 S_{1}\right] \operatorname{Li}_{2}(\eta)\right] \log (\eta) \\
& +\frac{8 \zeta_{2}}{9}+\frac{(\eta+1)\left(5 \eta^{2}+22 \eta+5\right)}{9 \eta^{3 / 2}}\left[6-8 S_{1}\right] \operatorname{Li}_{3}\left(\eta_{1}\right) \\
& +\frac{\left(\eta_{1}+1\right)^{2}\left(-10 \eta^{3 / 2}+5 \eta^{2}+42 \eta-10 \eta_{1}+5\right)}{36 \eta^{3 / 2}}\left[4 S_{1}-3\right] \operatorname{Li}_{3}(\eta) \\
& +\left(\frac{8\left(405 \eta^{2}-3238 \eta+405\right)}{729 \eta}+\frac{128 \zeta_{3}}{27}-\frac{320 \zeta_{2}}{27}\right) S_{1}+\left(\frac{64 \zeta_{2}}{9}+\frac{1856}{81}\right) S_{2} \\
& \left.-\frac{640}{81} S_{3}+\frac{128}{27} S_{4}-\frac{32 \zeta_{3}}{9}-\frac{2 R_{3}}{243 N^{2}(N+1)^{2} \eta}\right\} .
\end{aligned}
$$


Here $\eta_{1}=\sqrt{\eta}$ and the polynomials $R_{i}$ read

$$
\begin{aligned}
R_{1}= & 15 \eta^{2} N^{4}+78 \eta N^{4}+15 N^{4}+30 \eta^{2} N^{3}+156 \eta N^{3}+30 N^{3}+25 \eta^{2} N^{2}+18 \eta N^{2}+25 N^{2} \\
& +10 \eta^{2} N+4 \eta N+10 N+32 \eta \\
R_{2}= & 1215 \eta^{2} N^{8}-1596 \eta N^{8}+1215 N^{8}+4860 \eta^{2} N^{7}-6384 \eta N^{7}+4860 N^{7}+8100 \eta^{2} N^{6} \\
& -25844 \eta N^{6}+8100 N^{6}+7290 \eta^{2} N^{5}-39348 \eta N^{5}+7290 N^{5}+3645 \eta^{2} N^{4}-20304 \eta N^{4} \\
& +3645 N^{4}+810 \eta^{2} N^{3}-140 \eta N^{3}+810 N^{3}+432 \eta N^{2}+288 \eta N+864 \eta \\
R_{3}= & 405 \eta^{2} N^{4}-532 \eta N^{4}+405 N^{4}+810 \eta^{2} N^{3}-1064 \eta N^{3}+810 N^{3}+405 \eta^{2} N^{2}-1012 \eta N^{2} \\
& +405 N^{2}+96 \eta N+288 \eta .
\end{aligned}
$$

The $N$ dependence is described by rational contributions and harmonic sums, while for the dependence on the mass-ratio also polylogarithms [38] arise.

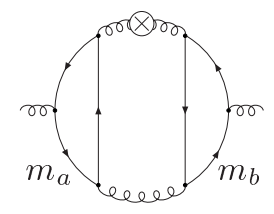

Figure 1: A typical scalar diagram contributing to the $\mathrm{OME} A_{g g, Q}^{(3)}$. It is symmetric under the change of the mass assignment $m_{a} \leftrightarrow m_{b}$. The diagram has been drawn using Axodraw [39].

Next we consider the contributions due to unequal masses for the gluonic operator matrix element $A_{g g, Q}^{(3)}$. We have obtained the $x$ - and Mellin space representations for all contributing scalar diagrams. The calculation of the Feynman diagrams is performed as follows. The Feynman parameter integrals can be carried out by introducing an intermediary Mellin-Barnes representation [40]. The corresponding sum of residues does usually not converge in the complete integration region for the Feynman parameter integrals. In order to ensure convergence, several transformations of the integrals have to be performed. The integration domain of the final integral is then split into two regions for each of which a convergent sum of residues can be obtained. The sums are solved applying the algorithms encoded in the package Sigma [16,17], EvaluateMultiSums and SumProduction [27]. We map the final integral to obtain the form of a Mellin transform

$$
\mathbf{M}[f(x)](N)=\int_{0}^{1} d x x^{N} f(x)
$$

and rewrite the $x$-space representation $f(x)$ in terms of iterated integrals with argument $x$ by using techniques inspired by [41]. The result cannot be described within the class of hyperlogarithms only, but more general iterated integrals involving square-root valued integration kernels [35] occur, see also [42]. In the corresponding Mellin space expressions, finite nested binomial sums weighted by $\left(\begin{array}{c}2 k \\ k\end{array}\right)$ are emerging. Here also the function

$$
\begin{aligned}
L_{1}(\eta) & =\frac{1}{2} \int_{0}^{\eta} d x \frac{\sqrt{x}}{1-x} \ln ^{2}(x) \\
& =4\left\{\operatorname{Li}_{3}\left(\eta_{1}\right)-\operatorname{Li}_{3}\left(-\eta_{1}\right)+\ln \left(\eta_{1}\right)\left[\operatorname{Li}_{2}\left(-\eta_{1}\right)-\operatorname{Li}_{2}\left(\eta_{1}\right)\right]-\frac{1}{2} \ln ^{2}\left(\eta_{1}\right) \ln \left(\frac{1-\eta_{1}}{1+\eta_{1}}\right)\right.
\end{aligned}
$$




$$
\left.-2 \eta_{1}\left[1-\ln \left(\eta_{1}\right)+\frac{1}{2} \ln ^{2}\left(\eta_{1}\right)\right]\right\},
$$

is frequently observed.

In a final step we apply the computer algebra package HarmonicSums [43-46] to generate a difference equation for the Mellin transform of $f(x)$ and solve it by using the packages Sigma, EvaluateMultiSums and SumProduction. As an example we present the $N$-space result for the diagram in Figure 1

$$
\begin{aligned}
& I(N)= \\
& \left(m_{b}^{2}\right)^{-3+3 / 2 \varepsilon}\left[\frac{1+(-1)^{N}}{2}\right]\left\{-\frac{\eta+1}{24 \varepsilon \eta^{2}(N+1)}+\left[P_{8} \frac{1}{5760 \eta^{3} N(N+1)^{2}(N+2)}\right.\right. \\
& +\frac{1}{45} \frac{2^{-2 N-9}\left(\begin{array}{c}
2 N \\
N
\end{array}\right) P_{2}}{(\eta-1) \eta^{3}(N+1)^{2}(N+2)} \sum_{i_{1}=1}^{N} \frac{2^{2 i_{1}}\left(\frac{\eta}{-1+\eta}\right)^{i_{1}}}{\left(\begin{array}{c}
2 i_{1} \\
i_{1}
\end{array}\right)}-\frac{1}{45} \frac{2^{-2 N-8}\left(\begin{array}{c}
2 N \\
N
\end{array}\right)}{\eta^{2}(N+1)^{2}(N+2)} P_{4} \\
& +\frac{\left(\frac{\eta}{\eta-1}\right)^{N}}{11520(\eta-1) \eta^{2} N(N+1)^{2}(N+2)} P_{5}+\frac{(1-\eta)^{-N} P_{6}}{11520(\eta-1) \eta^{3} N(N+1)^{2}(N+2)}+\frac{S_{1}\left(\frac{1}{1-\eta}, N\right)}{360(N+1)}+\frac{S_{1}\left(\frac{\eta}{\eta-1}, N\right)}{360 \eta^{3}(N+1)} \\
& \left.-\frac{1}{45} P_{11} \frac{2^{-2 N-9}\left(\begin{array}{c}
2 N \\
N
\end{array}\right)}{(\eta-1) \eta(N+1)^{2}(N+2)} \sum_{i_{1}=1}^{N} \frac{2^{2 i_{1}}(1-\eta)^{-i_{1}}}{\left(\begin{array}{c}
2 i_{1} \\
i_{1}
\end{array}\right)}\right] \log ^{2}(\eta)+\left[-\frac{P_{7}}{5760 \eta^{3}(N+1)^{2}(N+2)}\right. \\
& +\frac{1}{45}(\eta+1) \frac{2^{-2 N-7}\left(\begin{array}{c}
2 N \\
N
\end{array}\right)}{\eta^{3}(N+1)^{2}(N+2)} P_{2}-\frac{1}{45} \frac{2^{-2 N-8}\left(\begin{array}{c}
2 N \\
N
\end{array}\right) P_{2}}{(\eta-1) \eta^{3}(N+1)^{2}(N+2)} \sum_{i_{1}=1}^{N} \frac{2^{2 i_{1}}\left(\frac{\eta}{-1+\eta}\right)^{i_{1}} S_{1}\left(\frac{-1+\eta}{\eta}, i_{1}\right)}{\left(\begin{array}{c}
2 i_{1} \\
i_{1}
\end{array}\right)} \\
& +\frac{1}{90}\left(\eta^{3}-1\right) \frac{1}{\eta^{3} N(N+1)^{2}(N+2)} S_{1}(N)+\frac{(1-\eta)^{-N}}{5760(\eta-1) \eta^{3} N(N+1)^{2}(N+2)} P_{6} S_{1}(1-\eta, N) \\
& -\frac{\left(\eta^{3}-1\right) S_{2}(N)}{180 \eta^{3}(N+1)}-\frac{\left(\frac{\eta}{\eta-1}\right)^{N}}{5760(\eta-1) \eta^{2} N(N+1)^{2}(N+2)} P_{5} S_{1}\left(\frac{\eta-1}{\eta}, N\right)+\frac{S_{1,1}\left(\frac{1}{1-\eta}, 1-\eta, N\right)}{180(N+1)} \\
& -\frac{1}{180} \frac{1}{\eta^{3}(N+1)} S_{1,1}\left(\frac{\eta}{\eta-1}, \frac{\eta-1}{\eta}, N\right) \\
& \left.-\frac{1}{45} P_{10} \frac{2^{-2 N-8}\left(\begin{array}{c}
2 N \\
N
\end{array}\right)}{(\eta-1) \eta(N+1)^{2}(N+2)} \sum_{i_{1}=1}^{N} \frac{2^{2 i_{1}}(1-\eta)^{-i_{1}} S_{1}\left(1-\eta, i_{1}\right)}{\left(\begin{array}{c}
2 i_{1} \\
i_{1}
\end{array}\right)}\right] \log (\eta) \\
& +\left[\frac{\left(27 \eta^{2}+10 \eta+27\right)}{5760 \eta^{5 / 2}(N+1)}-\frac{2^{-2 N-8}\left(\begin{array}{c}
2 N \\
N
\end{array}\right) P_{1}}{45 \eta^{5 / 2}(N+1)^{2}(N+2)}\right] L_{1}(\eta)-\frac{1}{45}(\eta-1) \frac{2^{-2 N-6}\left(\begin{array}{c}
2 N \\
N
\end{array}\right)}{\eta^{3}(N+1)^{2}(N+2)} P_{2} \\
& +\frac{2^{-2 N}\left(\begin{array}{c}
2 N \\
N
\end{array}\right) P_{2}}{11520(\eta-1) \eta^{3}(N+1)^{2}(N+2)} \sum_{i_{1}=1}^{N} \frac{2^{2 i_{1}}\left(\frac{\eta}{-1+\eta}\right)^{i_{1}}\left[S_{1,1}\left(\frac{-1+\eta}{\eta}, 1, i_{1}\right)-S_{2}\left(\frac{-1+\eta}{\eta}, i_{1}\right)\right]}{\left(\begin{array}{c}
2 i_{1} \\
i_{1}
\end{array}\right)} \\
& -\frac{1}{2880 \eta^{3} N(N+1)^{2}(N+2)} P_{9}+\frac{(\eta+1) P_{3} S_{1}(N)}{5760 \eta^{3}(N+1)^{2}(N+2)}+\frac{\left(\eta^{3}+1\right)}{180 \eta^{3} N(N+1)^{2}(N+2)}\left[S_{2}(N)-S_{1}^{2}(N)\right] \\
& +\frac{\left(\eta^{3}+1\right) S_{3}(N)}{180 \eta^{3}(N+1)}+\frac{(1-\eta)^{-N} P_{6}}{5760(\eta-1) \eta^{3} N(N+1)^{2}(N+2)}\left[S_{1,1}(1-\eta, 1, N)-S_{2}(1-\eta, N)\right] \\
& +\frac{\left(\frac{\eta}{\eta-1}\right)^{N} P_{5}}{5760(\eta-1) \eta^{2} N(N+1)^{2}(N+2)}\left[S_{1,1}\left(\frac{\eta-1}{\eta}, 1, N\right)-S_{2}\left(\frac{\eta-1}{\eta}, N\right)\right]+\frac{1}{180(N+1)}\left[S_{1}\left(\frac{1}{1-\eta}, N\right)\right. \\
& S_{1,1}(1-\eta, 1, N)-S_{1,2}\left(\frac{1}{1-\eta}, 1-\eta, N\right)+S_{1,2}\left(1-\eta, \frac{1}{1-\eta}, N\right)-S_{1,1,1}\left(1-\eta, 1, \frac{1}{1-\eta}, N\right) \\
& \left.-S_{1,1,1}\left(1-\eta, \frac{1}{1-\eta}, 1, N\right)\right]+\frac{1}{180 \eta^{3}(N+1)}\left[S_{1}\left(\frac{\eta}{\eta-1}, N\right) S_{1,1}\left(\frac{\eta-1}{\eta}, 1, N\right)\right. \\
& +S_{1,2}\left(\frac{\eta-1}{\eta}, \frac{\eta}{\eta-1}, N\right)-S_{1,2}\left(\frac{\eta}{\eta-1}, \frac{\eta-1}{\eta}, N\right)-S_{1,1,1}\left(\frac{\eta-1}{\eta}, 1, \frac{\eta}{\eta-1}, N\right)
\end{aligned}
$$




$$
\begin{aligned}
& \left.-S_{1,1,1}\left(\frac{\eta-1}{\eta}, \frac{\eta}{\eta-1}, 1, N\right)\right]+\frac{2^{-2 N}\left(\begin{array}{l}
2 N \\
N
\end{array}\right) P_{10}}{11520(\eta-1) \eta(N+1)^{2}(N+2)} \\
& \left.\times \sum_{i_{1}=1}^{N} \frac{2^{2 i_{1}}(1-\eta)^{-i_{1}}\left[S_{2}\left(1-\eta, i_{1}\right)-S_{1,1}\left(1-\eta, 1, i_{1}\right)\right]}{\left(\begin{array}{c}
2_{1} \\
i_{1}
\end{array}\right)}\right\}
\end{aligned}
$$

where the $P_{i}$ represent different polynomials in the variables $N$ and $\eta$. Additionally to harmonic sums [33,34], we also observe generalized harmonic sums [45, 47]

$$
S_{b, \vec{a}}(c, \vec{d}, N)=\sum_{k=1}^{N} \frac{c^{k}}{k^{b}} S_{\vec{a}}(\vec{d}, k), \quad S_{\emptyset}=1, \quad b, a_{i} \in \mathbb{N} \backslash\{0\}, \quad c, d_{i} \in \mathbb{R} \backslash\{0\} .
$$

The real numerator weights are partly rational functions of the ratio $\eta$. Furthermore, nested binomial sums over generalized sums contribute $[35,48,49]$. Usually, the expansion of the integrals like $I(N)$ in terms of $\eta \ll 1$ up to a finite order for general values of $N$ is not possible in general. However, if a fixed integer value is chosen for $N$, the expansion may be performed. Here one obtains the same result as calculating the corresponding moment using the code $\mathrm{Q} 2 \mathrm{e} / \mathrm{EXP}$ [14].

\section{Conclusions}

We calculated contributions of $O\left(T_{F}^{2} C_{F(A)}\right)$ to the gluonic massive operator matrix elements at 3loop order in QCD from graphs with two massive quark lines, both for equal and unequal heavy quark internal lines ${ }^{4}$. In the calculation of these diagrams the evaluation of Mellin-Barnes integrals usually requires to close the contour either to the left or the right, depending on the value of one of the Feynman parameters. Both in intermediary and the final results, nested finite binomial sums occur, weighted by harmonic sums in the equal mass case. For unequal masses also generalized finite harmonic sums are present, the letters of which are rational expressions of the mass ratio of the two quarks. For general values of the Mellin variable $N$ the expansion in the mass ratio is not straightforward. One is rather advised to deal with the case of general mass ratios. The present summation technologies allow to compute the complete result, however.

\section{References}

[1] I. Bierenbaum, J. Blümlein and S. Klein, Nucl. Phys. B 820 (2009) 417 [arXiv:0904.3563 [hep-ph]].

[2] M. Buza, Y. Matiounine, J. Smith, R. Migneron and W. L. van Neerven, Nucl. Phys. B 472 (1996) 611 [hep-ph/9601302].

[3] A. Behring, I. Bierenbaum, J. Blümlein, A. De Freitas, S. Klein and F. Wißbrock, arXiv:1403.6356 [hep-ph].

[4] J. Ablinger, J. Blümlein, S. Klein, C. Schneider and F. Wißbrock, Nucl. Phys. B 844 (2011) 26 [arXiv:1008.3347 [hep-ph]].

[5] J. Ablinger, A. Behring, J. Blümlein, A. De Freitas, A. Hasselhuhn, A. von Manteuffel, M. Round, C. Schneider and F. Wißbrock, arXiv: 1406.4654 [hep-ph], Nucl. Phys. B (2014) in press.

[6] J. Ablinger et al., DESY 13-232, to be published.

\footnotetext{
${ }^{4}$ The case of one vanishing quark mass has been dealt with in Refs. $[4,50]$ before.
} 
[7] J. Ablinger, J. Blümlein, A. De Freitas, A. Hasselhuhn, A. von Manteuffel, M. Round, C. Schneider and F. Wißbrock, Nucl. Phys. B 882 (2014) 263 [arXiv:1402.0359 [hep-ph]].

[8] M. Buza, Y. Matiounine, J. Smith and W. L. van Neerven, Eur. Phys. J. C 1 (1998) 301 [hep-ph/9612398];

[9] I. Bierenbaum, J. Blümlein and S. Klein, Phys. Lett. B 672 (2009) 401 [arXiv:0901.0669 [hep-ph]].

[10] J. Blümlein and W. L. van Neerven, Phys. Lett. B 450 (1999) 417 [hep-ph/9811351].

[11] J. Blümlein and F. Wißbrock, DESY 14-019.

[12] J. Ablinger, J. Blümlein, S. Klein, C. Schneider and F. Wißbrock, arXiv:1106.5937 [hep-ph].

[13] J. Ablinger, J. Blümlein, A. Hasselhuhn, S. Klein, C. Schneider and F. Wißbrock, PoS RADCOR2011 (2011) 031 [arXiv:1202.2700 [hep-ph]].

[14] R. Harlander, T. Seidensticker and M. Steinhauser, Phys. Lett. B 426 (1998) 125 [hep-ph/9712228]. T. Seidensticker, hep-ph/9905298.

[15] J. Ablinger, J. Blümlein, A. De Freitas, A. Hasselhuhn, A. von Manteuffel, M. Round and C. Schneider, Nucl. Phys. B 885 (2014) 280 [arXiv:1405.4259 [hep-ph]].

[16] C. Schneider, Sém. Lothar. Combin. 56 (2007) 1, article B56b.

[17] C. Schneider, Computer Algebra in Quantum Field Theory: Integration, Summation and Special Functions Texts and Monographs in Symbolic Computation eds. C. Schneider and J. Blümlein (Springer, Wien, 2013) 325 arXiv:1304.4134 [cs.SC].

[18] M. Karr 1981 J. ACM 28 (1981) 305.

[19] C. Schneider, Symbolic Summation in Difference Fields Ph.D. Thesis RISC, Johannes Kepler University, Linz technical report 01-17 (2001).

[20] C. Schneider, J. Differ. Equations Appl. 11 (2005) 799.

[21] C. Schneider, J. Algebra Appl. 6 (2007) 415.

[22] C. Schneider, J. Symbolic Comput. 43 (2008) 611 [arXiv:0808.2543].

[23] C. Schneider, Appl. Algebra Engrg. Comm. Comput. 21 (2010) 1.

[24] C. Schneider, Motives, Quantum Field Theory, and Pseudodifferential Operators (Clay Mathematics Proceedings Vol. 12, eds. A. Carey, D. Ellwood, S. Paycha and S. Rosenberg (Amer. Math. Soc) (2010), 285 arXiv:0808.2543.

[25] C. Schneider, Ann. Comb. 14 (2010) 533 [arXiv:0808.2596].

[26] C. Schneider, in : Lecture Notes in Computer Science (LNCS) eds. J. Guitierrez, J. Schicho, M. Weimann, in press, arXiv:1307.7887 [cs.SC] (2013).

[27] J. Ablinger, J. Blümlein, S. Klein and C. Schneider, Nucl. Phys. Proc. Suppl. 205-206 (2010) 110 [arXiv:1006.4797 [math-ph]];

J. Blümlein, A. Hasselhuhn and C. Schneider, PoS RADCOR 2011 (2011) 032 [arXiv:1202.4303 [math-ph]];

C. Schneider, J. Phys. Conf. Ser. 523 (2014) 012037 [arXiv:1310.0160 [cs.SC]].

[28] C. Schneider, Advances in Applied Math 34(4) (2005) 740;

J. Ablinger, J. Blümlein, M. Round and C. Schneider, PoS LL 2012 (2012) 050 [arXiv:1210.1685

[cs.SC]];

M. Round et al., in preparation. 
[29] C. Studerus, Comput. Phys. Commun. 181 (2010) 1293.

[30] A. von Manteuffel and C. Studerus, arXiv:1201.4330 [hep-ph].

[31] R.H. Lewis, Computer Algebra System Fermat, http://home.bway . net/lewis .

[32] C. W. Bauer, A. Frink and R. Kreckel, Symbolic Computation 33 (2002) 1, cs/0004015 [cs-sc].

[33] J.A.M. Vermaseren, Int. J. Mod. Phys. A 14 (1999) 2037 [arXiv:hep-ph/9806280].

[34] J. Blümlein and S. Kurth, Phys. Rev. D 60 (1999) 014018 [arXiv:hep-ph/9810241].

[35] J. Ablinger, J. Blümlein, C. G. Raab and C. Schneider, arXiv:1407.1822 [hep-th].

[36] R. Tarrach, Nucl. Phys. B 183 (1981) 384;

O. Nachtmann and W. Wetzel, Nucl. Phys. B 187 (1981) 333;

N. Gray, D. J. Broadhurst, W. Grafe and K. Schilcher, Z. Phys. C 48, (1990) 673;

D. J. Broadhurst, N. Gray and K. Schilcher, Z. Phys. C 52 (1991) 111;

J. Fleischer, F. Jegerlehner, O. V. Tarasov and O. L. Veretin, Nucl. Phys. B 539 (1999) 671 [Erratum-ibid. B 571 (2000) 511] [arXiv:hep-ph/9803493];

S. Bekavac, A. Grozin, D. Seidel and M. Steinhauser, JHEP 0710 (2007) 006 [arXiv:0708.1729 [hep-ph]].

[37] L. F. Abbott, Nucl. Phys. B 185 (1981) 189;

A. Rebhan, Z. Phys. C 30 (1986) 309;

F. Jegerlehner and O. V. Tarasov, Nucl. Phys. B 549 (1999) 481 [arXiv:hep-ph/9809485].

[38] L. Lewin, Polylogarithms and Associated Functions, (North Holland, New Yourk, 1981).

[39] J.A.M. Vermaseren, Comput. Phys. Commun. 83 (1994) 45.

[40] E.W. Barnes, Proc. Lond. Math. Soc. (2) 6 (1908) 141; Quart. Journ. Math. 41 (1910) 136; H. Mellin, Math. Ann. 68 (1910) 305.

[41] F.C.S. Brown, Commun. Math. Phys. 287 (2009) 925 [arXiv:0804.1660 [math.AG]].

[42] U. Aglietti and R. Bonciani, Nucl. Phys. B 698 (2004) 277 [hep-ph/0401193];

R. Bonciani, G. Degrassi and A. Vicini, Comput. Phys. Commun. 182 (2011) 1253 [arXiv:1007.1891 [hep-ph]].

[43] J. Ablinger, arXiv:1011.1176 [math-ph].

[44] J. Ablinger, J. Blümlein and C. Schneider, J. Math. Phys. 52 (2011) 102301 [arXiv:1105.6063 [math-ph]].

[45] J. Ablinger, J. Blümlein and C. Schneider, J. Math. Phys. 54 (2013) 082301 [arXiv:1302.0378 [math-ph]].

[46] J. Ablinger, arXiv:1305.0687 [math-ph].

[47] S. Moch, P. Uwer and S. Weinzierl, J. Math. Phys. 43 (2002) 3363 [arXiv:hep-ph/0110083].

[48] J. Ablinger, J. Blümlein and C. Schneider, J. Phys. Conf. Ser. 523 (2014) 012060 [arXiv:1310.5645 [math-ph]].

[49] J. Ablinger, J. Blümlein, C. Raab, C. Schneider and F. Wißbrock, Nucl. Phys. B 885 (2014) 409 [arXiv:1403.1137 [hep-ph]].

[50] A. Behring, J. Blümlein, A. De Freitas, T. Pfoh, C. Raab, M. Round, J. Ablinger, A. Hasselhuhn, C. Schneider, and F. Wißbrock, PoS RADCOR 2013 (2014) 058 [arXiv:1312.0124 [hep-ph]]. 